\title{
Der wahre Preis für ein Schnitzel
}

\author{
Der ökologische Landbau gilt vielfach als ein Schlüssel für eine umwelt- \\ gerechtere Landnutzung. Die Bundesregierung hat sich deshalb zum Ziel \\ gesetzt seinen Flächenanteil bis zum Jahr 2010 auf 20 Prozent zu erhöhen. \\ Verursacht der ökologische Landbau tatsächlich weniger Umweltschäden als \\ die konventionelle Landwirtschaft? Wie hoch sind die externen Kosten beider \\ Produktionssysteme? Eine aktuelle Studie des IÖW beantwortet diese \\ Fragen am Beispiel der Schweinemast in Deutschland.
}

$\mathrm{J}$ Von Thomas Korbun, Michael Steinfeldt und Niels Koblschütter eder Einwohner in Deutschland verzehrte im Jahr 2003 40,3 Kilogramm Schweinefleisch, das machte rund zwei Drittel des gesamten Fleischkonsums aus (1). Dafür wurden 37,5 Millionen Schweine geschlachtet, nur 115.000 davon wurden ökologisch gemästet. Als Grundlage für eine Ökobilanz der beiden Produktionssysteme wurden Modellbetriebe definiert, die die Haltungsbedingungen der Mehrzahl der in Deutschland gemästeten Schweine repräsentieren: Konventionelle Schweine werden in der Regel in zwangsbelïfteten, beheizten Ställen auf Spaltenböden ohne Einstreu gehalten. Oft erreichen die Bestände 1.000 Tiere und mehr. Öko-Schweine werden in offenen, unbeheizten Ställen mit Einstreu gehalten. Repräsentativ sind 50 Tiere, die doppelt so viel Platz haben wie ihre konventionellen Artgenossen. Die Fütterung erfolgt mit Futtermitteln aus ökologischem Landbau, bis auf zugesetztes konventionelles Kartoffeleiweiß, was aber 2005 generell verboten sein wird. .Neben einem herkömmlichen Betrieb, der den verbreiteten Stand der Technik abbildet, wurde jeweils ein fortschrittlicher Betrieb betrachtet, der bessere Techniken zur Minderung von Umweltschäden einsetzt, der aber noch wenig verbreitet ist. Für jeden der vier Modellbetriebe wurden Daten über relevante Umweltwirkungen der Vorproduktion (Erzeugung von Düngemitteln, Pestiziden und Saatgut), des Futtermittelanbaus, der Futtermittelaufbereitung (einschließlich der Transporte), der Mast sowie der Gülle- beziehungsweise Mistlagerung und -ausbringung zusammengefuihrt und auf ein Kilogramm Schweinefleisch bezogen.

\section{- Ergebnisse der Ökobilanz}

Die konventionellen Modellbetriebe weisen einen 34 bis 41 Prozent höheren Energieaufwand auf. Für die Produktion von einem Kilogramm kon- ventionellem Schweinefleisch werden mehr als 100 Gramm Mineraldünger und 1,4 Gramm Pflanzenschutzmittel eingesetzt. Die ökologische Herstellung verzichtet auf beide Betriebsmittel. Der Stickstoff-Eintrag in Gewässer ist bei den konventionellen Betrieben mit rund 20 Gramm pro Kilogramm Schweinefleisch etwa viermal höher als bei den ökologischen.

Das Treibhauspotenzial bei der Erzeugung konventionellen Schweinefleischs ist etwa vier Mal höher als bei ökologischer Produktion. Dazu tragen vor allem die Produktion von Mineraldünger, der Futtermittelanbau, der Sojatransport und die Mast bei. Bei anderen Wirkungskategorien sind die konventionellen Betriebe besser: Das Versauerungspotenzial der ökologischen Betriebe ist rund 16 Prozent, das terrestrische Eutrophierungspotenzial rund 20 Prozent höher als bei den konventionellen. Das liegt wesentlich an den höheren Emissionen während der Mast, wo zum Beispiel Ammoniak aus den offenen Ställen der Öko-Schweine ungehindert entweichen kann. Das höchste Minderungspotenzial liegt hier aber im Technikeinsatz: Die beiden fortschrittlichen Betriebe weisen jeweils nur die Hälfte des Versauerungspotenzials der herkömmlichen Betriebs des gleichen Systems auf.

\section{Ökonomische Bewertung}

Die aus den Umweltschäden resultierenden Kosten spiegeln sich nicht in den Preisen für das Schweinefleisch wider. Nicht die Verursacher, also Produzenten oder Konsumenten, kommen für die Schäden auf, sondern die Allgemeinheit. In der Studie wurden diese externen Kosten anhand des Vermeidungskostenansatzes berechnet. Die Frage lautet: Welche Kosten müssten an anderer Stelle aufgewendet werden, um die negativen externen Effekte ,wieder gutzumachen“, also beispielsweise nitratverunreinigtes Grundwasser aufzuberei- ten. Das jeweils beste System wurde auf Null gesetzt. Die Vermeidungskosten für ein Kilogramm Schweinefleisch aus einem konventionellen Standard-Betrieb erreichen 47,3 Cent. Das macht etwa ein Drittel des Erzeugerpreises aus, den der Landwirt vom Abnehmer erhält. Der herkömmliche ökologische Betrieb verursacht mit 14,5 Cent nur weniger als ein Drittel dieser externen Kosten. Auch der fortschrittliche konventionelle Betrieb erreicht mit 33,7 Cent noch einen erheblich höheren Betrag als der fortschrittliche ökologische Betrieb mit 1,6 Cent.

\section{Fazit}

Mit der Studie wurde erstmals eine produktbezogene ökologisch-ökonomische Bewertung der Schweinemast vorgelegt. Die ökologische Produktionsweise verursacht danach erheblich geringere externe Kosten durch Umweltschäden als die konventionelle. Neben den im Rahmen der Studie monetarisierten Umweltwirkungen sprechen noch weitere Aspekte für die ökologische Schweineproduktion: etwa die tiergerechtere Haltungsform, die zu weniger Krankheiten und Verhaltensschäden führt, oder der Verzicht auf gentechnisch veränderte Futtermittel und auf den vorbeugenden Einsatz von Antibiotika.

\section{Anmerkung}

(1) Die Studie wurde im Auftrag von foodwatch e.V. durchgeführt und von der Stiftung für Bildung und Behindertenförderung (SBB) gefördert. Vgl. auch Korbun, T. et al.: Was kostet ein Schnitzel wirklich? Ökologisch-ökonomischer Vergleich der konventionellen und der ökologischen Produktion von Schweinefleisch in Deutschland. IÖW-Schriftenreihe 171/04, Berlin 2004. Download Kurzfassung: www.foodwatch.de/cmsimages/1080128187foodwatch-

Schnitzelreport_240304.pdf

\section{Die Autoren}

Thomas Korbun ist Wissenschaftlicher Geschäftsführer des Instituts für ökologische Wirtschaftsforschung (IÖW). Michael Steinfeldt ist wissenschaftlicher Mitarbeiter im Forschungsfeld Ökologische Unternehmenspolitik am IÖW. Niels Kohlschütter arbeitet am Institut für Organischen Landbau (IOL) der Universität Bonn.

Kontakt: IÖW, Potsdamer Str. 105, 10785 Berlin. Tel. 030-8845940, E-Mail: thomas.korbun@ioew.de 
(c) 20I0 Authors; licensee IÖW and oekom verlag. This is an article distributed under the terms of the Creative Commons Attribution Non-Commercial No Derivates License (http://creativecommons.org/licenses/by-nc-nd/3.o/), which permits unrestricted use, distribution, and reproduction in any medium, provided the original work is properly cited. 